\title{
Participation of CCL1 in Snail-Positive Fibroblasts in Colorectal Cancer Contribute to 5-Fluorouracil/Paclitaxel Chemoresistance
}

Ziqian Li, $\mathrm{PhD}^{1}$
Kaying Chan, $\mathrm{MS}^{1}$
Yifei Qi, $\mathrm{PhD} \mathrm{D}^{1,2}$
Linlin Lu, $\mathrm{PhD}^{1}$
Fen Ning, $\mathrm{PhD}^{3}$
Mengling Wu, MPharm ${ }^{1}$
Haifang Wang, $\mathrm{PhD}^{1}$
Yuan Wang, $\mathrm{PhD}^{1}$
Shaohui Cai, $\mathrm{PhD}^{4}$
Jun Du, $\mathrm{PhD}^{1}$

${ }^{1}$ Department of Microbial and Biochemical Pharmacy, School of Pharmaceutical Sciences, Sun Yat-sen University, Guangzhou, China, ${ }^{2}$ Markey Cancer Center, University of Kentucky, Lexington, KY, USA,

${ }^{3}$ Department of Obstetrics, Guangzhou Institute of Pediatrics, Guangzhou Women and Children's Medical Center, Guangzhou Medical University, Guangzhou, ${ }^{4}$ Department of Pharmacology, School of Pharmaceutical Sciences, Jinan University, Guangzhou, China

\begin{abstract}
Purpose
Cancer-associated fibroblasts (CAFs) activated by cancer cells has a central role in development and malignant biological behavior in colorectal cancer (CRC). Adult fibroblasts do not express Snail, but Snail-positive fibroblasts are discovered in the stroma of malignant $\mathrm{CRC}$ and reported to be the key role to chemoresistance. However, the reciprocal effect of CAFs expressed Snail to chemoresistance on CRC cells and the underlying molecular mechanisms are not fully characterized.
\end{abstract}

\section{Materials and Methods}

Snail-overexpressed 3T3 stable cell lines were generated by lipidosome and CT26 mixed with 3T3-Snail subcutaneous transplanted CRC models were established by subcutaneous injection. Cell Counting Kit-8, flow cytometry and western blotting assays were performed, and immunohistochemistry staining was studied. The cytokines participated in chemoresistance was validated with reverse transcriptase-polymerase chain reaction and heatmap.

\section{Results}

Snail-expression fibroblasts are discovered in human and mouse spontaneous CRCs. Overexpression of Snail induces 3T3 fibroblasts transdifferentiation to CAFs. CT26 co-cultured with 3T3-Snail resisted the impairment from 5-fluorouracil and paclitaxel in vitro. The subcutaneous transplanted tumor models included 3T3-Snail cells develop without restrictions even after treating with 5-fluorouracil or paclitaxel. Moreover, these chemoresistant processes may be mediated by CCL1 secreted by Snail-expression fibroblasts via transforming growth factor $\beta /$ nuclear factor-kB signaling pathways.

\section{Conclusion}

Taken together, Snail-expressing 3T3 fibroblasts display CAFs properties that support 5-fluorouracil and paclitaxel chemoresistance in CRC via participation of CCL1 and suggest that inhibition of the Snail-expression fibroblasts in tumor may be a useful strategy to limit chemoresistance.
Correspondence: Jun Du, $\mathrm{PhD}$

Department of Microbial and Biochemical Pharmacy, School of Pharmaceutical Sciences, Sun Yat-sen University, No. 132 Waihuan East Road, University Town, Guangzhou 510006, China Tel/Fax: 86-020-39943022

E-mail: dujun@mail.sysu.edu.cn

Received July 27, 2017

Accepted September 13, 2017

Published Online September 18, 2017

\section{Key words}

Colorectal neoplasms, Cancer-associated fibroblasts, Snail, Multidrug resistance, CCL1 


\section{Introduction}

Colorectal cancer $(\mathrm{CRC})$ is the third most common cancer and the second leading causal factor of cancer-related deaths worldwide [1]. Systemic chemotherapy, based on 5-fluorouracil, is the first-line therapy for patients with unresectable CRCs. However, the entire treatment effects on quite a lot of CRC patients are very limited, mainly due to drug resistance [2]. Since the cancer microenvironment is critical to the biological behavior of the tumor, elucidating the role of cancer-associated fibroblasts (CAFs), which is the most abundant cell-type in the matrix [3], is likely to associate with the chemoresistance in tumors. However, the chemoresistance function of fibroblasts in CRC is still unclear. A better understanding of the fibroblasts involved in chemoresistance on CRC should assist with the development of more efficient therapeutic methods.

Fibroblasts are usually in the quiescent state and become activated in the wound healing response [4]. In the activation process, fibroblasts differentiate into more active connective tissue cells called myofibroblasts, which produce more collagen than their precursors [5]. Myofibroblasts, induced by transforming growth factor $\beta$ (TGF- $\beta$ )-mediated signalling, express $\alpha$-smooth muscle actin ( $\alpha$-SMA) stress fibers [6]. Therefore, $\alpha$-SMA is the special marker of CAFs. CAFs are a major component of stroma in cancer, which have cemented themselves as key components of cancer progression [7]. A relentless clinical challenge for cancer therapy is the development of resistance and CAFs have emerged as key players in promoting cancer cell evasion of anticancer therapies.

Chemokine C-C motif ligand-1 (CCL1), interacting exclusively with the chemokine (C-C motif) receptor 8 (CCR8), is an inflammatory mediator [8]. Studies showed that CCL1 induced tumor cells metastasis into lymph nodes [9]. In addition, CCL1 exerts anti-apoptotic and proliferative activity in cancer cells which were treated with chemotherapy drugs [10]. Thence, like numerous other chemokines, CCL1 goes far beyond the effects of inflammation.

Snail, belonging to the zinc finger transcription repressor superfamily, is described as a TGF- $\beta$ target and the key regulator of epithelial-mesenchymal transition (EMT) [11]. There is acknowledged that adult fibroblasts do not express Snail, but Snail-positive fibroblasts were discovered in activated mesenchymal cells and in the stroma of malignant CRC [12]. Moreover, Snail was recently implicated in the regulation of both immunosuppression and immune tolerance [13]. As a consequence, these findings suggest the potential role of Snail in regulating cancer-related fibroblasts. However, the phenomenon of scattered Snail-expressing fibroblasts imposing chemoresistance on cancer cells is poorly defined, and no links between Snail activity and the genera- tion of chemoresistance have been proposed. Here, by using Snail-related stable 3T3 cell lines together with CT26 murine CRC cell lines, we demonstrated that Snail-expressing fibroblasts are the critical factors in the chemoresistance on CRC.

\section{Materials and Methods}

\section{Establishment of mice primary colorectal tumor model and cell culture}

The primary CRC models used were based on the previously described [14].

Mouse colon cancer cells (CT26) and mouse fibroblast cells (NIH3T3) were purchased from the Type Culture Collection of the Chinese Academy of Sciences (Shanghai, China). Cells were cultivated in Dulbecco's modified Eagle's medium (DMEM) medium and supplemented with $10 \%$ fetal bovine serum (FBS), $100 \mu \mathrm{g} / \mathrm{mL}$ streptomycin and $100 \mathrm{U} / \mathrm{mL}$ penicillin in a moistened atmosphere at $37^{\circ} \mathrm{C}$ where was enriched with $5 \% \mathrm{CO}_{2}$.

\section{Generation of 3T3-pldSnail cells}

The murine Snail expression vector was constructed for generation of Snail overexpression cell line. Briefly, the murine Snail gene was cloned into pL-tdTomato-Neo, a 2A peptide-linked vector which was received by Dr. Changmin Chen from Harvard Medical School, to generate the red fluorescent protein plasmid pL-tdTomato-mSnail. Snail and resistance genes were two $2 \mathrm{~A}$ peptide genes linked together in a row [15]. $3 \mathrm{~T} 3$ cells were seeded onto a 6 -well plate $\left(1.5 \times 10^{5}\right.$ cells/well) before transfection, allowing the confluency to grow to $80 \%-90 \%$. Liposomemediated transfection was used to introduce the pL-tdTomato-mSnail plasmid into 3T3. The expression of the red fluorescence protein was used to monitor the transfection effciency. G418 $(800 \mu \mathrm{g} / \mathrm{mL})$ was applied to selecting the transfected 3T3 cells for 10-14 days. Picked out and sowed into 96-well plate, the survived cells were cultured for cell clones formation and further expansion. After expanded, the monoclonal cell populations were named 3T3Snail, respectively.

The mock cells transfected with pL-tdTomatoNon was named as 3T3-mock. The morphous and the fluorescence intensity of the stable cells were observed through the inverted fluorescence microscope. 


\section{Histological study}

The CRC tissue from patient is from the First Affiliated Hospital of Clinical Medicine of Guangdong Pharmaceutical University in Huizhou. In brief, the colorectal tissues sections were harvested and fixed in $10 \%$ formalin for 2 days. The fixed tissues were sliced into $4 \mu \mathrm{m}$ after being embedded into paraffin. Firstly, the slices were deparaffinized and hydrated. Highpressure antigen retrieval was performed in citric acid buffer (pH 6.0). Endogenous peroxidase was blocked using $3 \%$ hydrogen peroxide for 20 minutes. And then, the slices were incubated with normal goat serum to block non-specific staining. Rabbit anti-Snail (1:200, ab53519, Abcam, Cambridge, MA), rabbit anti- $\alpha$-SMA (1:200, BS8796, Bioworld Technology, St. Louis Park, MN) and rabbit anti-cleaved caspase-3 (1:200, ab13847, Abcam) were used to incubate with the sections overnight at $4^{\circ} \mathrm{C}$. After washing by PBST, the sections were hatched with horseradish peroxidase (HRP) anti-rabbit secondary antibody. After staining with diaminobenzidine kit, the sections were counterstained with hematoxylin. Negative control was subjected to perform simultaneously, and phosphate buffered saline (PBS) was replaced of the primary antibody. Finally, the stained slices were observed under the microscopy.

\section{Co-culture}

CT26 cells were plated in a 6-well plate and 3T3-Snail cells were on 6-well Millicell Hanging Cell Culture Insert (Millicell, Sigma, St. Louis, MO) with $0.8-\mu \mathrm{m}$ pore size. Cells were co-cultured for 1 day whereas cells on the impermeable support were treated with 5-fluorouracil/paclitaxel or dimethyl sulfoxide for 2 days before quantification of CT26 live cells.

\section{Conditioned medium preparation}

Fibroblasts were cultivated in DMEM containing with $10 \%$ FBS for 24 hours. The conditioned medium (CM) was collected and the cellular debris were removed by filtration with a $0.22-\mu \mathrm{m}$ filter (Merck Millipore, Bedford, MA). The CM can be kept in $4^{\circ} \mathrm{C}$ within 48 hours.

\section{Cell death analysis}

Cell death of CT26 with fibroblasts CM or non-fibroblasts CM was evaluated by the Cell Counting Kit-8 (CCK-8; Dojindo, Kumamoto, Japan). In short, cells in complete medium were seeded into 96-well plates with 5,000 cells per well. After 12 hours, the cells were cultured with $30 \%$ or $60 \%$ fibroblasts $\mathrm{CM}$ with 5-fluorouracil or paclitaxel treatment. And 48 hours later, $10 \mu \mathrm{L}$ CCK-8 solution was added into each well and the plates were incubated at $37^{\circ} \mathrm{C}$ for 3 hours.
The cell viability was measured at $450 \mathrm{~nm}$ by scanning with microplate reader.

\section{Flow cytometric analyses}

Cleaved caspase-3 (ab13847, Abcam) and goat anti-rabbit IgG-fluorescein isothiocyanate (FITC) (ab6717, Abcam) were used to detect apoptosis rate. CT26 mixed with 3T3-mock/ 3T3-Snail was collected in eppendorf and incubated with anti-cleaved caspase-3 in room temperature for half an hour. After washing by PBS, anti-rabbit-FITC was used to combine with cleaved caspase-3. Then the expression of cleaved caspase-3 in CT26 was measured by flow cytometric analysis according to the procedure. The apoptotic rate was shown as histogram.

\section{Establishment of subcutaneous transplanted colorectal tumor model}

The 4-5-week-old female BALB/c mice were purchased from the Animal Experimental Center of Sun Yat-sen University (Guangzhou, China). The procedures for the experimental handling and care for the mice were approved by the Animal Experimentation Ethics Committee of Sun Yat-sen University (Guangzhou). CT26 and fibroblasts were mixed at a ratio of 1:2. Until the tumor volumes grew to approximately $100 \mathrm{~mm}^{3}$, treatment with 5-fluorouracil or paclitaxel was began. The first day of the treatment was designated as day 1 . The dose of 5 -fluorouracil was $30 \mathrm{mg} / \mathrm{kg}$, tail vein injection every 3 days. Paclitaxel was injected $10 \mathrm{mg} / \mathrm{kg}$ into the veins of the tail. The 5-fluorouracil/paclitaxel dose used in mice was based on the conversion formula of the drug concentration between human and animals. Subcutaneous tumor volumes were measured daily by a caliper. The tumor volumes calculation formula: tumor volume $=0.5$ length $\times$ width $\times$ width.

\section{Western blotting}

The total protein fractions in the cells were lysed by RIPA buffer (Beyotime Institute of Biotechnology, Jiangsu, China) with phenylmethylsulfonyl fluoride $(0.1 \mathrm{mM})$ and centrifuged at $12,000 \mathrm{rpm} / \mathrm{min}$ for 20 minutes. The supernatants were collected and quantified by BCA Protein Assay Kit (Beyotime Biotechnology). The proteins were separated electrophoretically by sodium dodecyl sulphate polyacrylamide gel electrophoresis and transferred onto polyvinylidene difluoride (Millipore, Billerica, MA). And then the 5\% nonfat dried milk was used to block the non-specific binding for 120 minutes at room temperature. Subsequently the membranes were incubation with primary antibodies (final dilution, 1:1,000, rabbit polyclonal, all provided from Cell Signa- 
ling Technology, Beverly, MA) in primary antibody diluent overnight at $4^{\circ} \mathrm{C}$. After washing, the membranes were incubated with HRP-conjugated anti-rabbit (final dilution, 1:5,000, Bioworld Technology) secondary antibodies in 5\% non-fat dried milk at room temperature for $120 \mathrm{~min}$. Washed by PBST three times for 30 minutes, the immunoreactivity and signals of the membranes were monitored by Chemiluminescence Reagent (Life Science, Inc., Boston, MA) in Tanon 5200 Multi instrument (Shanghai, China).

\section{Gene expression heatmap}

The total RNA was extracted by Trizol (Invitrogen, Carlsbad, CA), quantitative real-time polymerase chain reaction was performed by Prime Script TM RT reagent Kit (R036A, Takara, Tokyo, Japan) and SYBR Premix Ex Taq II (R820A, Takara). The program was running for 2 minutes at $95^{\circ} \mathrm{C}$, following by 40 cycles of 5 seconds at $95^{\circ} \mathrm{C}$ and 30 seconds at $58^{\circ} \mathrm{C}, 5$ seconds at $95^{\circ} \mathrm{C}$ and 5 seconds at $65-95^{\circ} \mathrm{C}$ (in $0.5^{\circ} \mathrm{C}$ increase). The general principles of primers were designed in the GeneBank database and the sequences were as follows: CCL1 (gene ID: NC_000077.6) forward primer, 5'-GGCTGAACAAAGGTAGAGAAAGC-3'; CCL1 reverse primer, 5'-TGGAGGACTGAGGGAAACTG-3'; TNF $\alpha$ (gene ID: NC_ 000083.6) forward primer, $5^{\prime}$-CACCACCATCAAGGACTCAA-3'; TNF $\alpha$ reverse primer, 5'-GAGACAGAGGCAACCTGACC-3'; myc (gene ID: NC_000081.6) forward primer, 5'-CTGTGGAGAAGAGGCAAACC-3'; myc reverse primer, 5'-TTGTGCTGGTGAGTGGAGAC-3'; CCL11 (gene ID: NC_ 000077.6) forward primer, $5^{\prime}$-GAGAGCCTACAGAGCCCAGA-3'; CCL11 reverse primer, 5' ${ }^{\prime}$-ACCGTGAGCAGCAGGAATAG-3'; CCL21 (gene ID: NC_000009.12) forward primer, 5'-CCAACTCACAGGCAAAGAGG-3'; CCL21 reverse primer, 5'-GCCAGGTAAGAAAGGGATGG-3'; CXCL1 (gene ID: NC_000071.6) forward primer, $5^{\prime}$-ACCCAAACCGAAGTCATAGC-3'; CXCL1 reverse primer, 5'-ACAGGTGCCATCAGAGCAGT-3'; IL17a (gene ID: NC_000067.6) forward primer, $5^{\prime}$-TCTCTATGGTCAGCGTTCCA-3'; IL17a reverse primer, $5^{\prime}$-AGGTGGTTTAGGAGGGCAAG-3'; matrix metalloproteinase (MMP) 9 (gene ID: NC_000068.7) forward primer, 5'-TCACTTTCCCTTCACCTTCG -3'; MMP9 reverse primer, 5'-TGCCGTCCTTATCGTAGTCA-3'; MMP23 (gene ID: NC_000070.6) forward primer, 5'-GGGACCACTTCAACCTCACA-3'; MMP23 reverse primer, 5'-GGAAACATCACTCCACATTCG-3'; glyceraldehyde 3-phosphate dehydrogenase (GAPDH) (gene ID: NC_000072.6) forward primer, 5'-GAGTCAACGGATTTGGTCGT-3'; GAPDH reverse primer, 5'-GACAAGCTTCCCGTTCTCAG-3'. The threshold cycle $(\mathrm{Ct})$ values of cytokines, chemokines, interleukins and MMPs in each sample were normalized by the values of GAPDH. The relative fold changes in mRNA expression level were calculated with the comparative threshold cycle
(Ct) method. The fold-change differences were constructed in a heatmap with sample clustering using OmicShare Platform.

\section{Statistical analysis}

Five biological replicates were used in mouse studies and all other studies utilized three biological replicates. All data were expressed as mean $\pm \mathrm{SD}$. In short, the unpaired twotailed Student's t test or two-way ANOVA test was analyzed by IBM SPSS Statistics ver. 20 (IBM Corp., Armonk, NY) to compare differences between different treatment groups. A $p$-value $<0.05$ was considered as statistically significant.

\section{Ethical statement}

The animal procedures were consistent with the guidelines of Sun Yat-sen University Institutional Animal Care and Use Committee.

The patient donated the colorectal tumor tissue and signed the informed consent which outlined the application and purpose of the research. The proposal was approved by the Ethical Committee of School of Pharmaceutical Sciences, Sun Yat-set University under the Chinese Ethical Regulations.

\section{Results}

\section{Snail expresses in the CAFs of human CRC tissues and Kunming mice's spontaneous CRCs}

$\alpha$-SMA is a common myofibroblast marker and can be used to appraisal CAFs. The identification of the fibroblasts is verified as previously described using Masson staining (Wanleibio, Shenyang, China) (Fig. 1A and D) and $\alpha$-SMA (1:200, Bioworld) (Fig. 1B and E). To investigate the Snail expression in CAFs, the antibody to Snail was used to immunohistochemical staining (Fig. 1C and F). Statistical analysis showed that in addition to cancer cells, Snail also express in CAFs both in the human CRC tissue (Fig. 1A-C) and Kunming mice CRC tissue (Fig. 1D-F). The results indicated that CAFs-rich Snail had something to do with the development and progression of CRC in all probability.

\section{Fibroblasts, overexpressed Snail, display CAFs proper- ties whose conditioned media increases proliferation of CRC cells}

To testify the function of Snail-expressing CAFs on CRC cells, the stable Snail overexpression 3T3 fibroblast (3T3- 

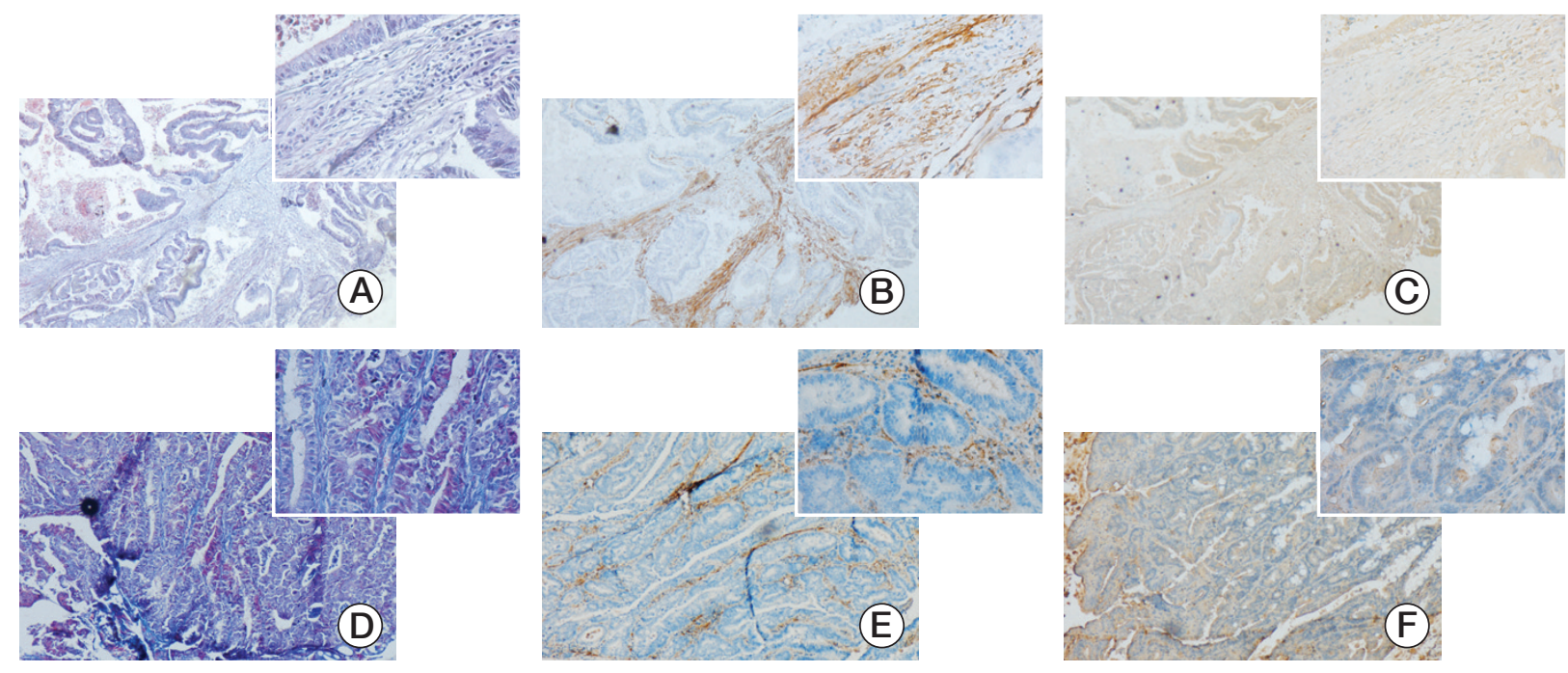

Fig. 1. Expression of Snail in cancer associated fibroblasts of human colorectal cancer tissues and Kunming (KM) mice's spontaneous colorectal cancers. (A, D) Masson staining of paraffin-embedded colorectal cancer tissue sections $(\times 40)$ and the figure in the top right corner was enlarged $(\times 400)$. (B, E) Activated fibroblast marker $\alpha$-smooth muscle actin expression in paraffin-embedded colorectal cancer tissue sections was detected by immunohistochemistry. (C, F) Immunohistochemistry was used to detect the Snail expression. The paraffin-embedded colorectal cancer tissue sections of panels A, B and C were from human and panels D, E and F were KM mice's spontaneous colorectal cancers tissue sections. Besides tumor cells, cancer-associated fibroblasts expressed Snail.

Snail) was generated by pL-tdTomato-mSnail plasmid (S1 Fig.). Using these reporters, we found that $\alpha$-SMA was expressed at higher levels in 3T3-Snail stable cell line compared to the stable blank control cell line 3T3-mock (Fig. 2A). Given the CAFs properties of 3T3-Snail, we undertook to analyze their function during CRC cells development in vitro. Results showed that the CM without FBS of 3T3-Snail cell line can increase proliferation or mutually assure the survival of CT26 (Fig. 2B), indicating that Snail expression in fibroblasts was contributed to the most fundamental activity of cancer cells. Furthermore, if Snail-expression fibroblasts could still contribute to the survival of CT26 under the treatment of chemotherapeutics, we would say that Snail-expression fibroblasts induced chemoresistance in CT26.

\section{Snail-expressing fibroblasts induce 5-fluorouracil/pacli- taxel resistance in CRC cells in vitro}

To extend the chemoresistance role of 3T3-Snail in vitro, we used $30 \%$ or $60 \%$ ratio CM of 3T3-Snail to co-culture with CT26. The relative sensitivity to 5-fluorouracil, dividing cancer cells to cell death via thymineless death [16], and paclitaxel, one of several cytoskeletal drug that target tubulin [17], in CT26 were assessed by CCK-8 Kit (Dojindo). Notably, we noted that the $\mathrm{IC}_{50}$ to 5-fluorouracil and paclitaxel of CT26 co-cultured with 3T3-Snail CM went upper compared with the control ones (Fig. 3A). Caspase-3, a central role in the execution-phase of cell apoptosis, and poly(ADP-ribose) polymerase (PARP), the cleavage target protein of caspase, are responsible for chromatin condensation and DNA fragmentation, as known as typical role in apoptosis [18]. After treating with 5-fluorouracil or paclitaxel, the expressions of cleaved caspase- 3 and cleaved PARP in CT26 were increasing. In contrast, no significant expressions of cleaved caspase-3 and cleaved PARP were observed for CT26, co-cultured with 3T3-Snail indirectly (Fig. 3B). The increased expression of caspase- 3 and PARP might partly result from the stimulation of the apoptotic cells which may act in a compensatory fashion to combat the 5-fluorouracil or paclitaxel increasing demand for cells; but the mechanism remained to be further studied. Furthermore, directly co-culturing CT26 cells with 3T3-Snail cell lines, we separated CT26 from 3T3Snail and analyzed the expression of cleaved caspase- 3 in CT26 by using flow cytometric method (S2 Fig.). Similarly, the results testified that Snail-expression 3T3 fibroblasts induced 5-fluorouracil/ paclitaxel resistance in CT26 in vitro (Fig. 4). 

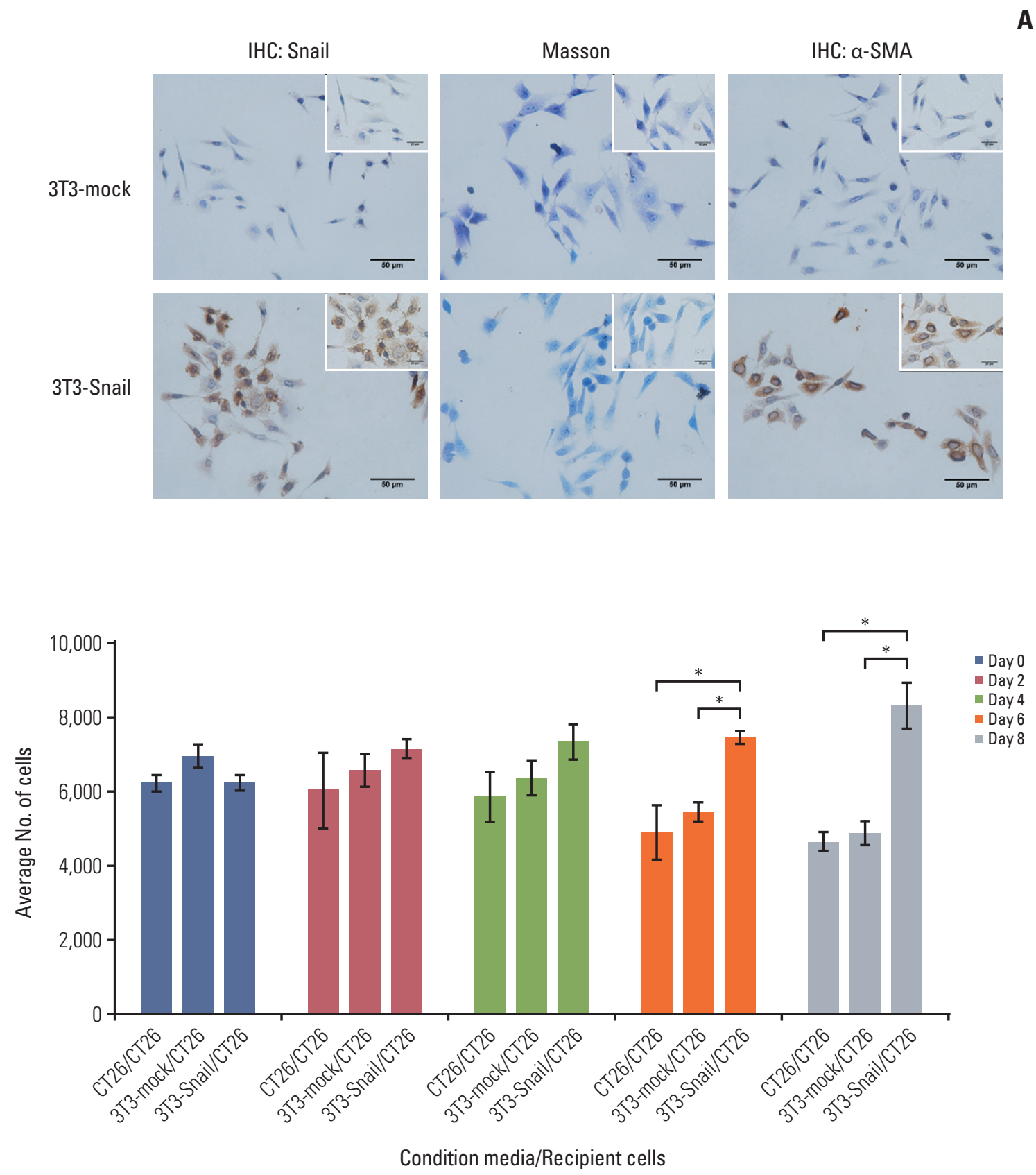

Fig. 2. Fibroblasts, overexpressed Snail, display cancer associated fibroblasts (CAFs) properties whose conditioned media ensures survival of colorectal cancer cells. (A) Snail and $\alpha$-smooth muscle actin ( $\alpha$-SMA) expressions in 3T3-mock and 3T3Snail stable cell lines grown on a coverslip were detected by immunohistochemistry (IHC) $(\times 400$; inset $\times 1,000)$. Masson staining of 3T3-mock and 3T3-Snail were also applied. 3T3-Snail stable cell lines expressed $\alpha$-SMA, present characteristics of CAFs. (B) CT26 cells were grown in 3T3-mock-conditioned media or 3T3-Snail-conditioned media without fetal bovine serum for 8 days and total cells were counted daily by Cell Counting Kit 8 . The conditioned media of 3T3-Snail guaranteed the survival of CT26 ( $\left.{ }^{*} \mathrm{p}<0.05\right)$. 

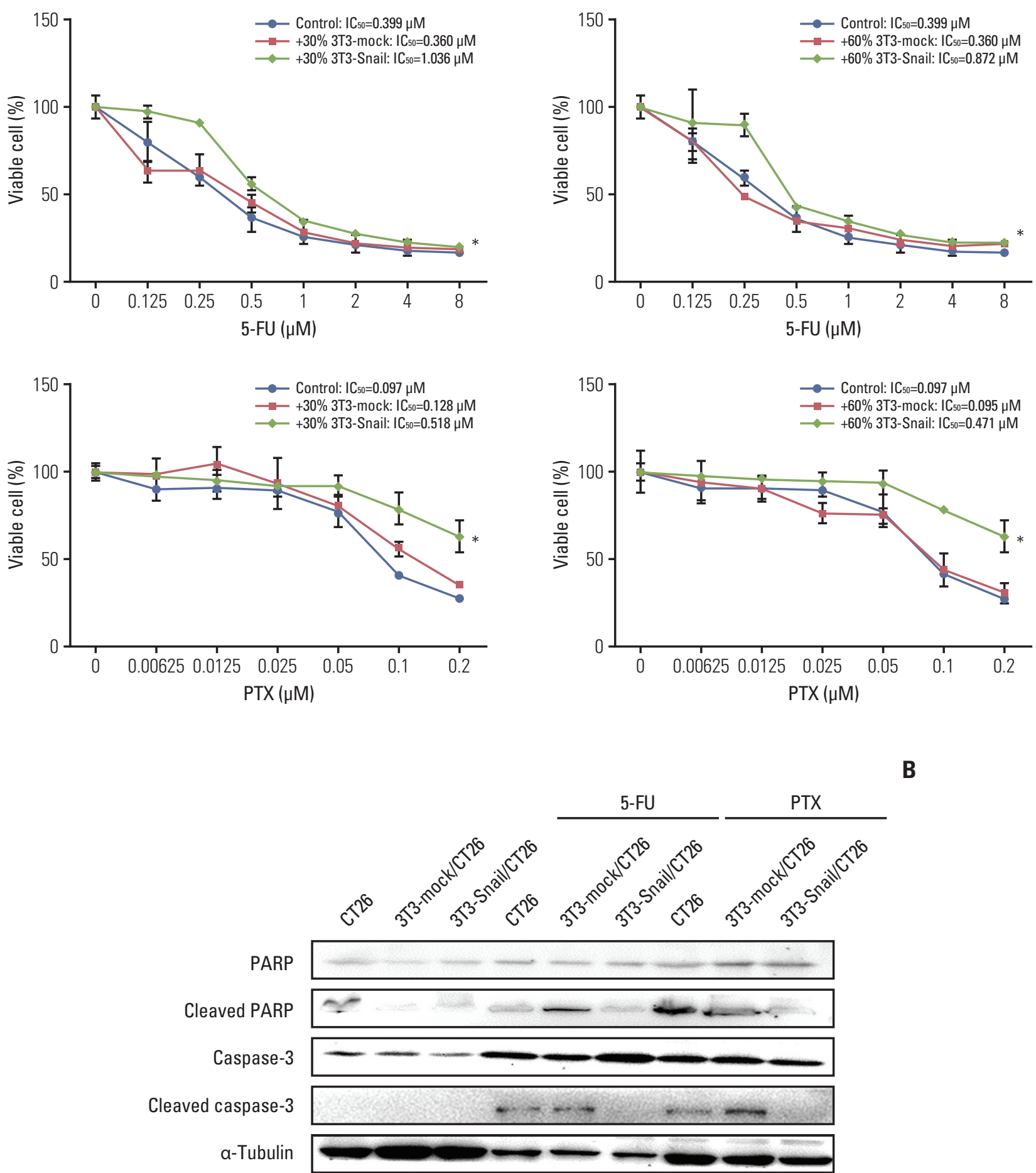

Fig. 3. Snail-overexpressed fibroblasts-conditioned media (CM) induce 5-fluorouracil (5-FU)/ paclitaxel (PTX) resistance in colorectal cancer cells in vitro. (A) Murine colorectal cancer CT26 cells were treated with the indicated dose of 5-FU/PTX alone or co-cultured with 30\% / 60\% 3T3-mock/3T3-Snail-conditioned media for 48 hours, and the cell viability was examined by Cell Counting Kit 8 assay. The IC $\mathrm{I}_{50}$ values of 5 -FU/PTX for CT26 were determined $\left({ }^{*} \mathrm{p}<0.05\right)$. (B) The levels of the poly(ADP-ribose) polymerase (PARP), cleaved PARP, caspase-3, and cleaved caspase- 3 proteins from CT26 alone or co-cultured with 3T3-mock/3T3-Snail CM were analyzed by Western blotting. 3T3-Snail CM contained something induced 5-FU/PTX resistance in CT26. 

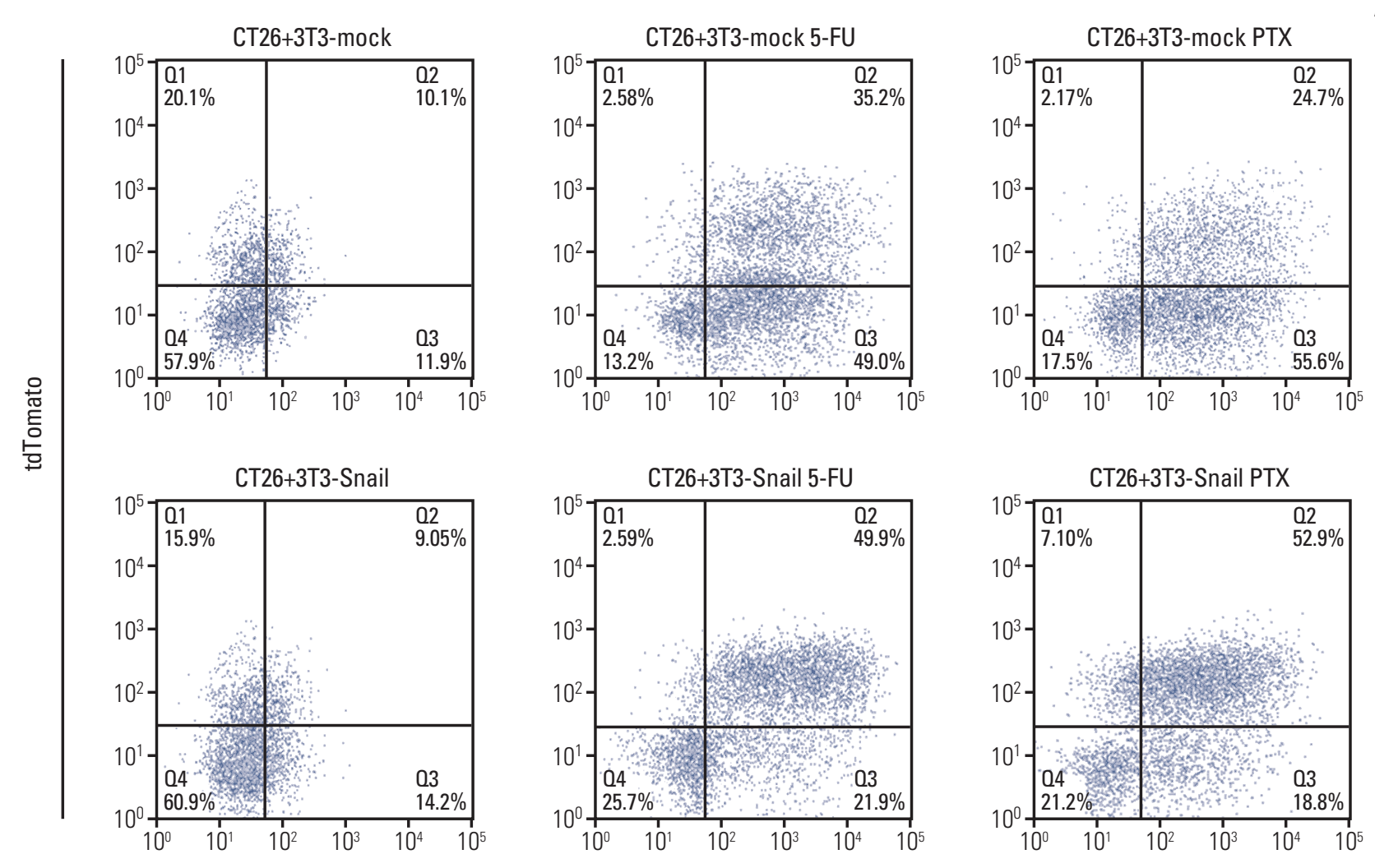

A
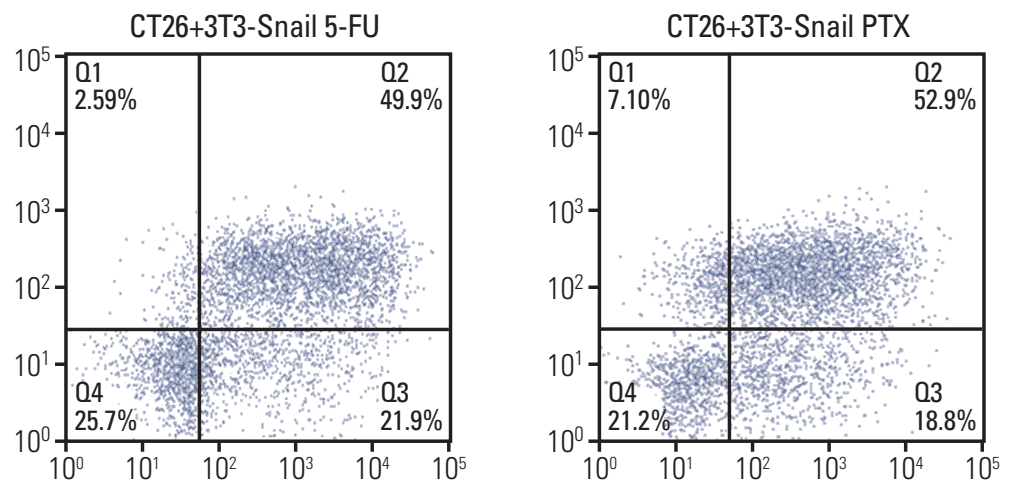

Cleaved caspase-3/FITC

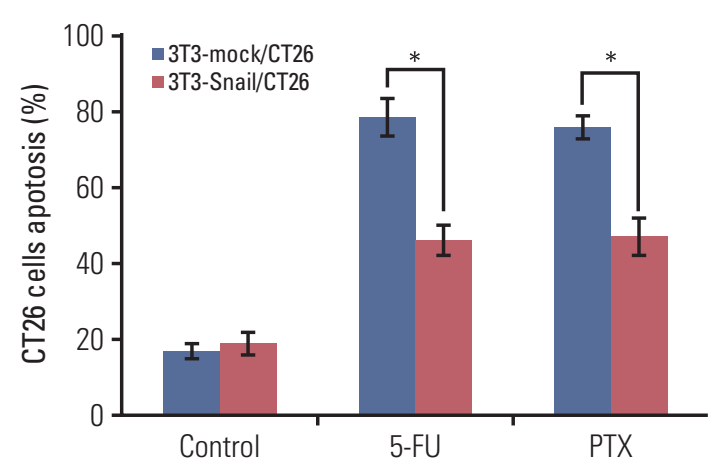

Fig. 4. Snail-overexpressed fibroblasts directly induce 5-fluorouracil (5-FU)/ paclitaxel (PTX) resistance in colorectal cancer cells in vitro. CT26 were pre-cultured with 3T3-mock or 3T3-Snail by 1:2 ratios for 24 hours and further treated with 5-FU $(0.8 \mu \mathrm{M}) /$ PTX $(0.2 \mu \mathrm{M})$ for an additional 48 hours. (A) Cells were collected and incubated with cleaved caspase-3-FITC, the percentage of cleaved caspase-3-FITC in CT26 cells was detected using flow cytometry. (B) Q3/ Q3+Q4) panel: quantifications of caspase- 3 at CT26 in a representative mammary gland $\left({ }^{*} \mathrm{p}<0.05\right)$. 3T3-Snail decreased CT26 cells apoptosis from 5-FU/PTX. 
Tumor inoculation 5-FU/PTX administration Tumor collection

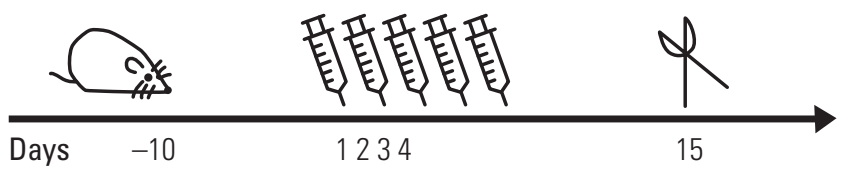

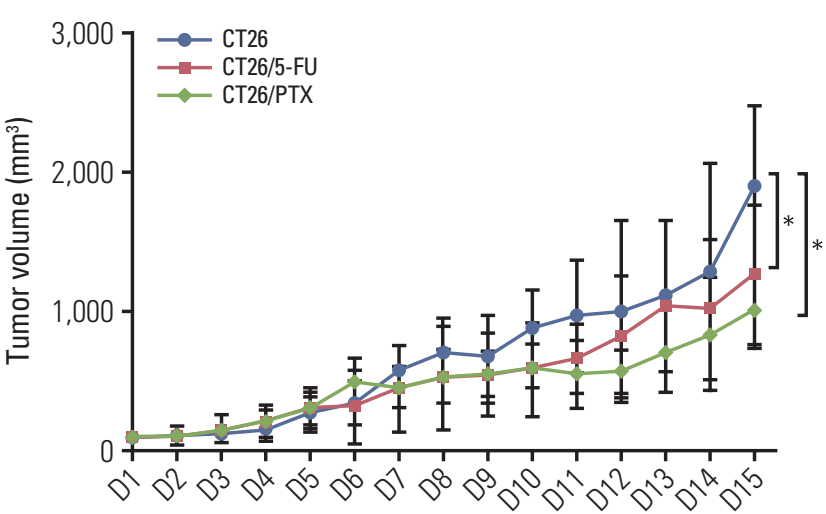

C

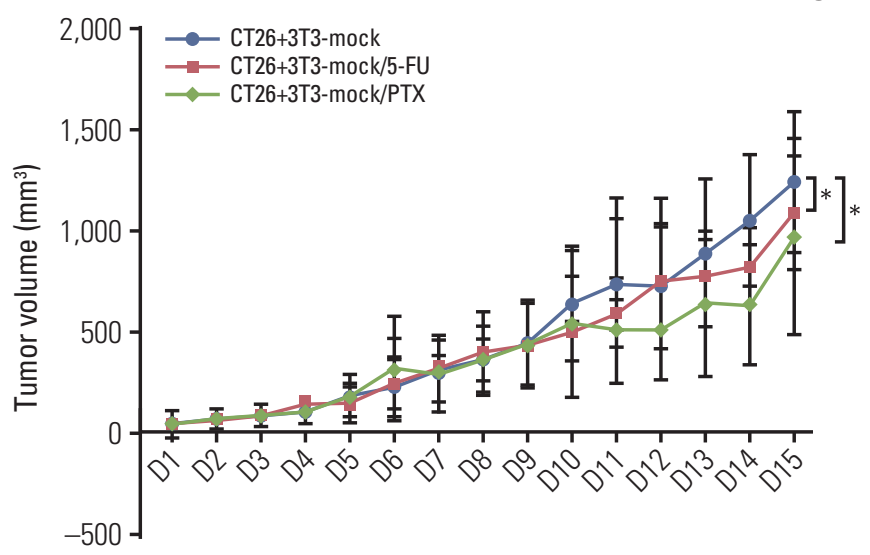

Fig. 5. Snail-overexpressed fibroblasts induce 5-fluorouracil (5-FU)/ paclitaxel (PTX) resistance in the subcutaneous transplanted in BABL/c mice. (A) Treatment protocol of scheduling administration of $5-\mathrm{FU}(30 \mathrm{mg} / \mathrm{kg}) / \mathrm{PTX}(15 \mathrm{mg} / \mathrm{kg})$ in tumorbearing mice by tail vein injection. $(\mathrm{B}, \mathrm{C})$ Tumor growth curves showed that tumors grew much slower in groups of tumor CT26 cells alone and CT26 cells mixed with 3T3-mock treated with 5-FU or PTX than the groups without treatment (n=5, $\left.{ }^{*} \mathrm{p}<0.05\right)$. (D) Meanwhile, tumor grew without any restriction in the groups of 3T3-Snail mixed with CT26 tumor cells.

\section{Snail-overexpressed fibroblasts induce 5-fluorouracil/ paclitaxel resistance in the subcutaneous transplanted in BALB/c mice}

To analyze whether 3T3-Snail induces chemoresistance behavior in an identical manner in vivo, we mixed the Snail up-regulation of 3T3 fibroblasts and CT26 cells at a ratio of 2:1 to establish the subcutaneous transplanted carcinoma. When tumor developed to approximately $100 \mathrm{~mm}^{3}$, the mice were treated with 5-fluorouracil or paclitaxel every 3 days and collected the tumor tissues after 15 days (Fig. 5A). Tumor dimensions were daily recorded and tumor growth curves were plotted. The results shown that 5-fluorouracil or paclitaxel resulted in decreased tumor growth of the CT26 groups and CT26 mixed with 3T3-mock groups (Fig. 5B and C). Nevertheless, the growth of the subcutaneous transplanted constructed by 3T3-Snail and CT26 was unrestricted, regardless of treating with 5-fluorouracil or paclitaxel (Fig. 5D). Masson dying and immunohistochemistry were used to assess the quantity of CAFs in tumor tissues; representative micrographs are presented in Fig. 6A. Overt $\alpha$-SMA staining was observed in the tumor tissue of 3T3-Snail groups and there was tiny minority in the other two groups (Fig. 6A). Furthermore, analyzing the expression of cleaved caspase- 3 in the tumor tissues, it showed the striking different expression of cleaved caspase-3 (Fig. 6B). Notably, although treating with 5-fluorouracil or paclitaxel, the expression of cleaved caspase-3 in 3T3-Snail and CT26 mixing tumor tissues was 

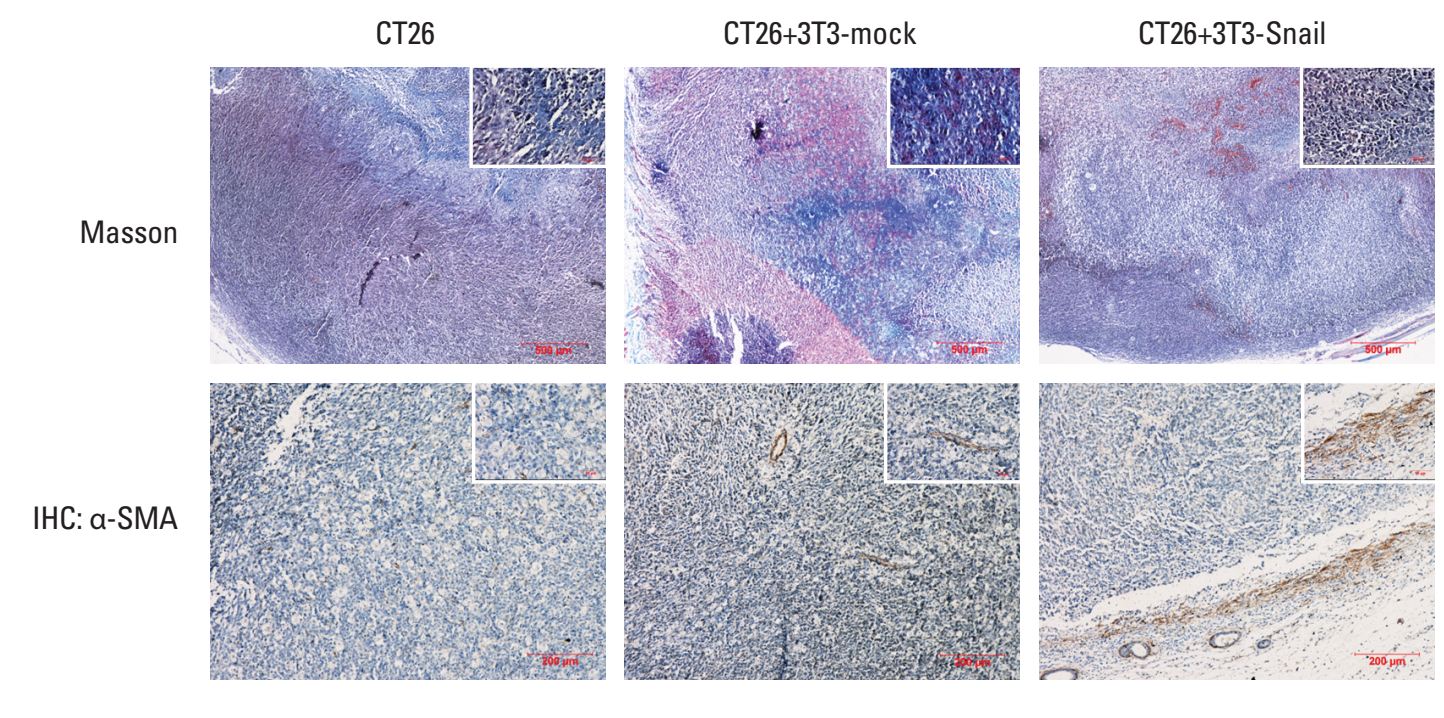

A
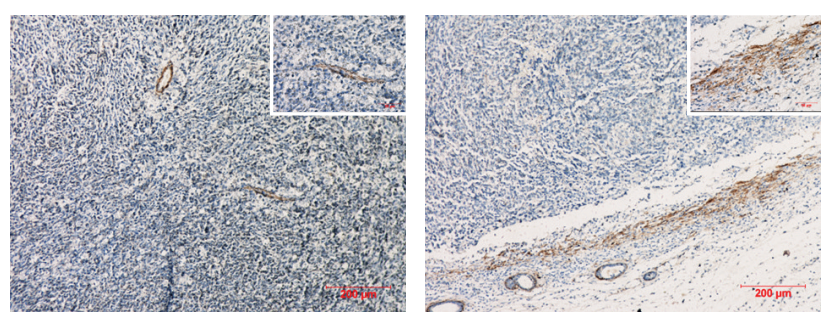

B
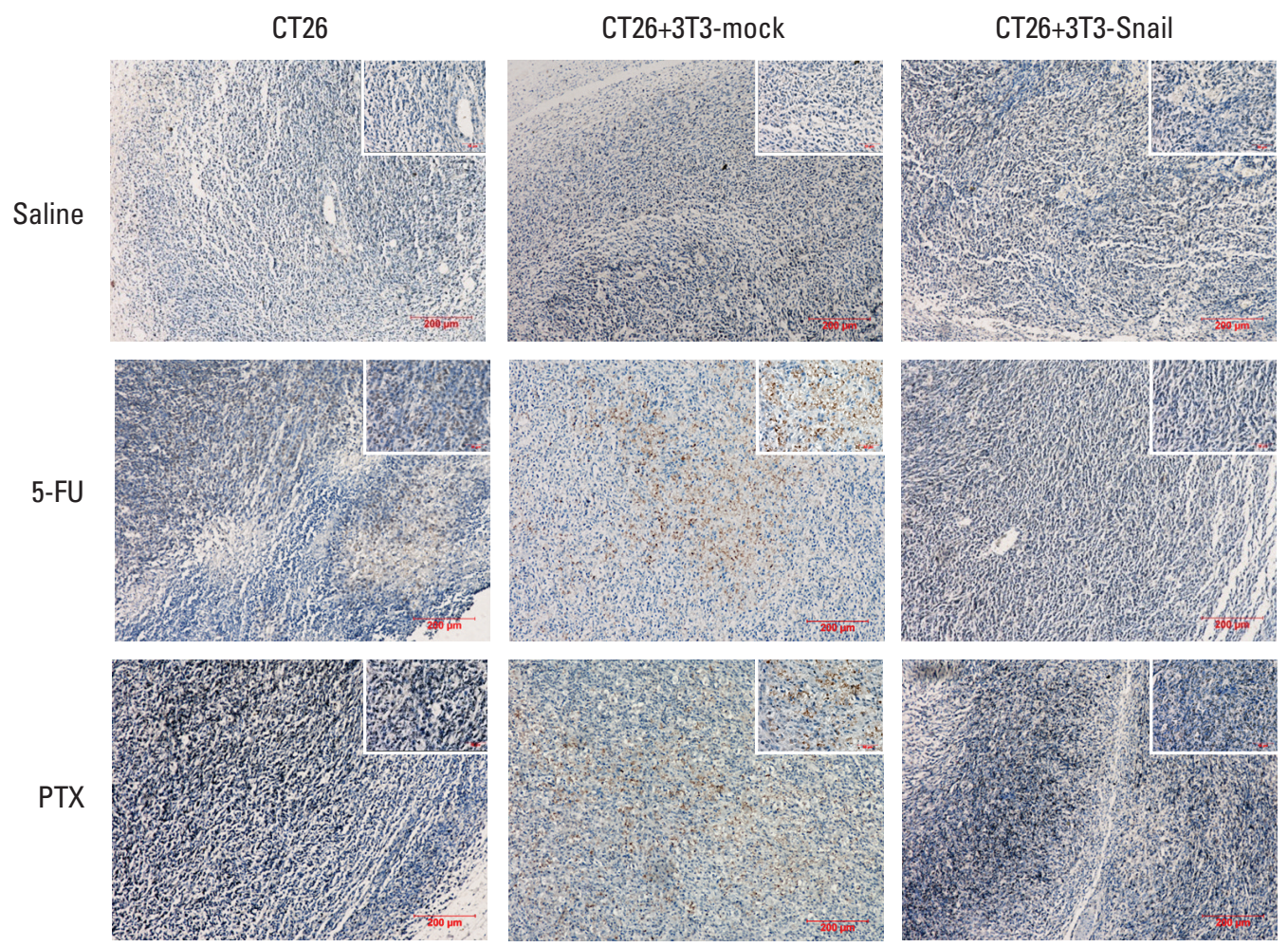

Fig. 6. Cleaved caspase-3 expression in the tumor tissue sections of subcutaneous transplanted in BALB/c mice. (A) Representative images of Masson-stained and $\alpha$-smooth muscle actin ( $\alpha$-SMA)-stained in paraffin sections of CT26 tumor tissue were shown. The tumor of CT26 mixed with 3T3-Snail expressed much more $\alpha$-SMA than other groups. (B) Representative images of cleaved caspase- 3 staining in paraffin sections of CT26 tumor tissue were shown. The expression of cleaved caspase-3 showed much less in the group of CT26 mixed with 3T3-Snail after treatment with 5-fluorouracil (5-FU)/ paclitaxel (PTX) than other groups. IHC, immunohistochemistry. 
A

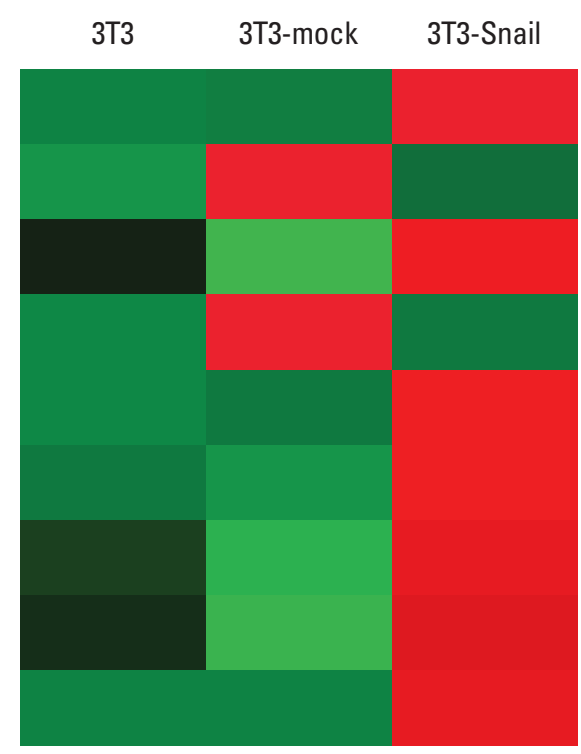

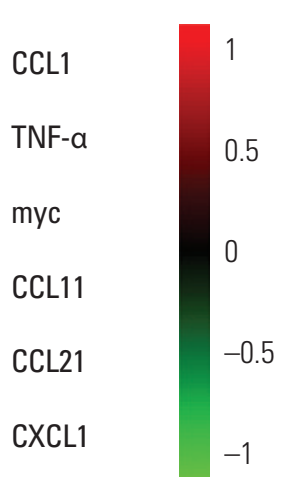

IL17a

MMP9

MMP23

Fig. 7. Role of chemokine C-C motif ligand-1 (CCL1) in 3T3-Snail contribute to chemoresistance in colorectal cancer. (A) Heatmap representation of RNA expression levels in 3T3, 3T3-mock, and 3T3-Snail. The data was shown graphically in visualized by using a heatmap tool, OmicShare Tool. (B) The protein level of multidrug resistance protein 1 (MDR1) in CT26 after co-cultured with 3T3-Snail was shown by Western blot analysis. MDR1 expressed increasedly in CT26 co-cultured with 3T3-Snail cell lines. (C) CT26 cells were treated with CCL1 $(100 \mathrm{ng} / \mathrm{mL})$ and/or transfected si-CCR8 for 48 hours and the expression of MDR1 was detected by Western blotting. CCR8, chemokine (C-C motif) receptor 8; IL, interleukin; MMP, matrix metalloproteinase.

lower than others. These findings also revealed that Snailexpression fibroblasts contribute to 5 -fluorouracil and paclitaxel resistance.

\section{Role of CCL1 in Snail-expressed 3T3 fibroblast con- tributes to chemoresistance in CRC}

By screening the expression of chemokine in 3T3-Snail, we found that the expression of CCL1 increased more notably than other chemokines (Fig. 7A). CCL1, interacting exclusively with the CCR8, exerts anti-apoptotic and proliferative activity [19]. Hence, we defined the functions of CCL1 in CRC and wanted to know whether CCL1 induced chemoresistance in CT26. Thereafter, CT26 were cultured with 3T3Snail and harvested 48 hours later. Western blotting results showed that the expression of multidrug resistance protein 1 (MDR1) in CT26 overtly increased (Fig. 7B). Furthermore, using CCL1 to treat CT26, the expression of MDR1 in CT26 also increased (Fig. 7C). However, the chemoresistance induced by CCL1 could be reversed by silencing the expression of CCR8 in CT26 (Fig. 7C).

\section{CCL1 possibly contributes to chemoresistance in CT26 via TGF- $\beta$ and nuclear factor $\kappa B$ signaling pathways}

To investigate whether TGF- $\beta$ and other related signaling pathways were involved in CCL1 induced chemoresistance in CT26, the total and phosphorylation levels of STAT3, GSK3 $\beta, \beta$-catenin, ERK, Smad2, AKT, and nuclear factor $\kappa B$ (NF-KB) were measured by western blotting. Our results revealed that CCL1 significantly phosphorylated ERK, Smad2, AKT, and NF-kB but not other molecules in CT26 (Fig. 8A). To ensure the role of TGF- $\beta$ and NF- $\mathrm{kB}$ signaling pathways in CCL1 induced chemoresistance, CT26 cells were pretreated with SB431542 (the inhibitor of TGF- $\beta$ signaling pathway) and BAY11-7081 (the inhibitor of NF- $\mathrm{kB}$ ) and then CCL1 was used to treat. The results revealed that both SB431542 and BAY11-7081 inhibited multidrug resistance (Fig. 8B). Furthermore, SB431542 reversed CCL1 induced ERK and Smad2 phosphorylation (Fig. 8C) and BAY11-7081 also reversed AKT and NF- $\mathrm{kB}$ phosphorylation (Fig. 8D). Strikingly, CCL1 may be tightly associated with Snail-expression fibroblasts induced tumor chemoresistance via TGF- $\beta$ and NF-kB signaling pathways. 

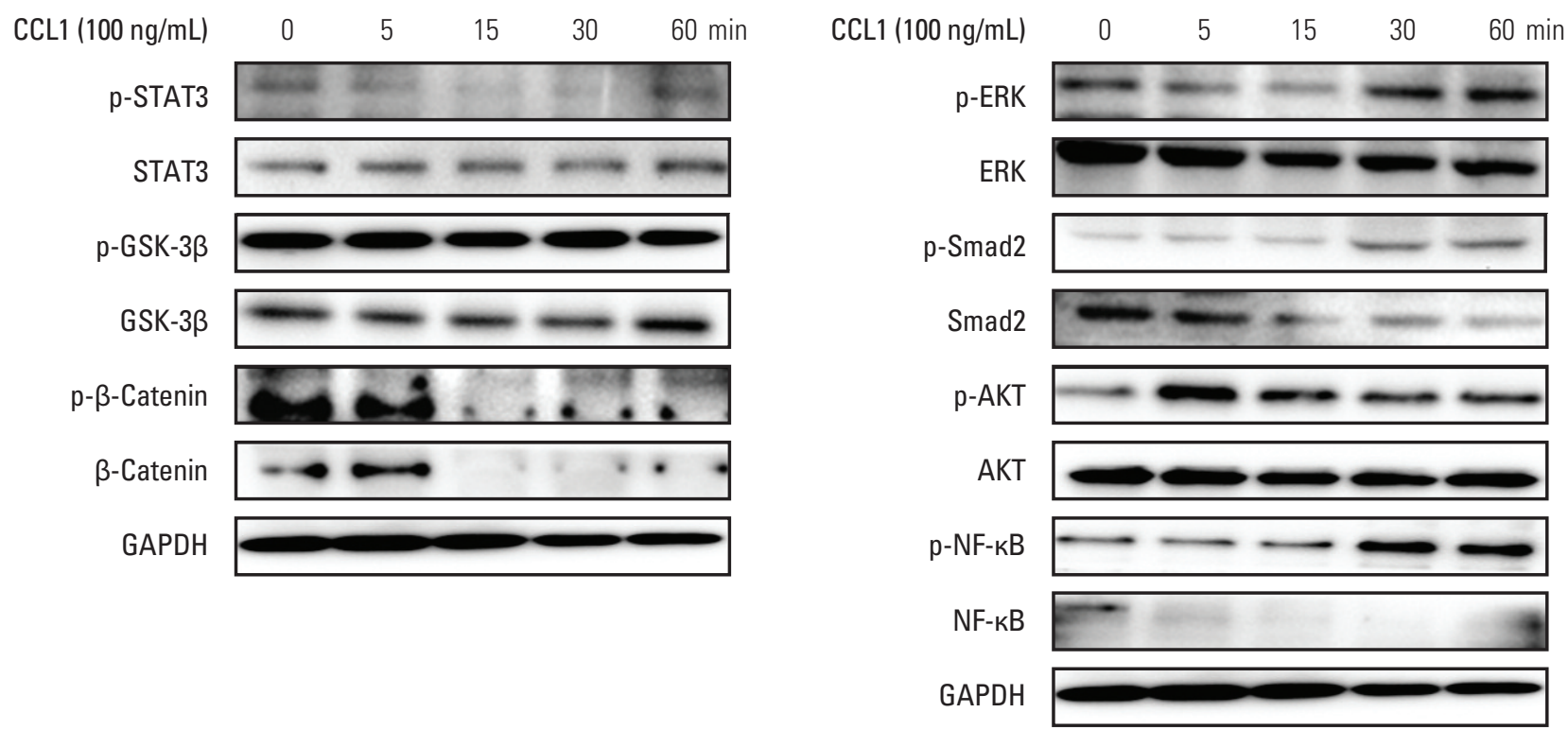

B
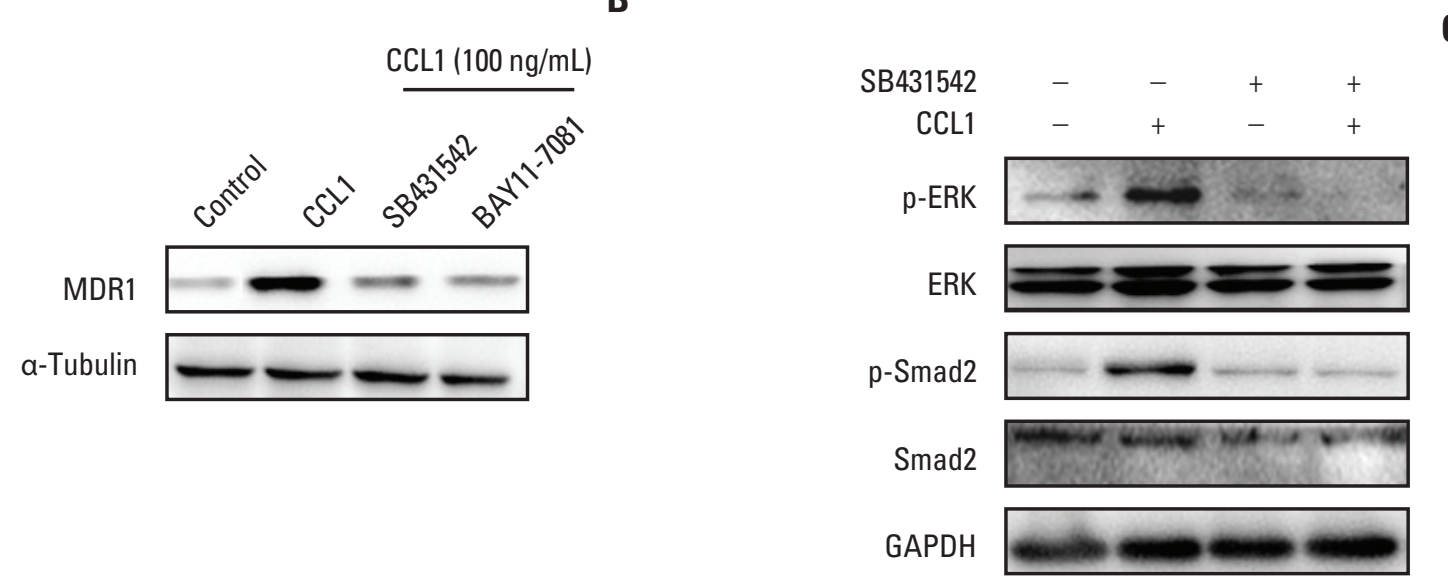

Fig. 8. Chemokine (C-C motif) ligand 1 (CCL1) contribute to chemoresistance in CT26 via transforming growth factor $\beta$ (TGF- $\beta$ ) and nuclear factor $\mathrm{\kappa B}$ (NF-кB) signaling pathways. (A) CT26 cells were treated with CCL1 for 5 minutes, 15 minutes, 30 minutes, and 1 hour, and the key proteins of several signaling pathways were detected via western blotting. (B) CT26 cells were pretreated with or without SB431542 $(20 \mu \mathrm{M})$, BAY11-7081 $(10 \mu \mathrm{M})$ for 2 hours and treated with CCL1 $(100 \mathrm{ng} / \mathrm{mL})$ for 48 hours, and then the expression of multidrug resistance protein 1 (MDR1) was analyzed by western blot. (C, D) Cells were pretreated with or without SB431542 $(20 \mu \mathrm{M})(\mathrm{C}) /$ BAY11-7081 $(10 \mu \mathrm{M})(\mathrm{D})$ for 2 hours and treated with or without CCL1 (100 ng / mL) for 5 minutes, and TGF- $\beta$, NF-кB pathway key proteins were analyzed by western blot. GAPDH, glyceraldehyde 3-phosphate dehydrogenase. (Continued to the next page) 


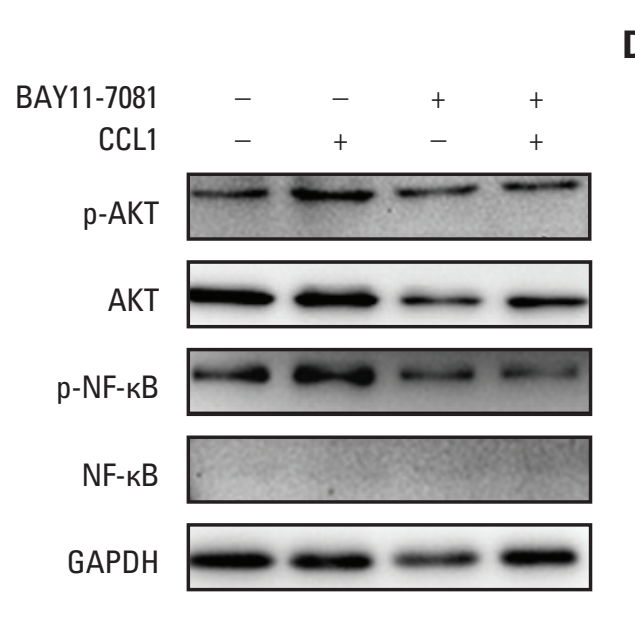

Fig. 8. (Continued from the previous page)

\section{Discussion}

Recently, chemotherapies did not significantly improve clinical outcomes of CRC patient, which is likely to medicines resistance. The interaction between cancer cells and tumor microenvironment is a very important factor in the regulation of cancer angiogenesis, invasion, metastasis and chemotherapy [20]. CAFs, as the major component of tumor stroma, are the key roles to chemoresistance in cancer cells [4]. There have been reported that CAFs could promote resistance to chemotherapy of many cancers. Hu et al. [21] demonstrated that CAFs contributed to chemoresistance in colon cancer by deriving exosomes. Besides, chemotherapy may induce the activation of CAFs to secrete cytokines such as interleukin 17 and contribute to resistance of chemotherapy [22]. Collectively, these results suggested that CAFs could enhance chemoresistance through induction various types of factors in CRC cells.

Snail, known as the trigger EMT, is reported significant association to patient survival when it expressed in tumor stroma [23]. Franci et al. [12] indicated that fibroblastic Snail expression predicted poor outcome of CRC. Here, we found that the CAFs in the human colon cancer tissue and KM mice's spontaneous CRC tissue expressed Snail. To indicate of the role of fibroblastic Snail in CRC, we generated the Snail-overexpressed 3T3 stable cell lines and found that Snail-expressing $3 \mathrm{~T} 3$ fibroblasts presented a characteristic of CAFs and increased proliferation of CT26. Multiple studies assessing CAFs indicated that CRC chemoresistance is increased by CAFs [24]. Analogously, we demonstrated a significant correlation between Snail-overexpression in 3T3 and the 5-fluorouracil/ paclitaxel resistance in vitro. In order to further strengthen the prognostication impact of Snailexpressing fibroblasts for chemoresistance in CRC, we developed the subcutaneous transplanted of CRC model by using CT26 and 3T3-Snail in the ratio of 1:2. After treating of 5-fluorouracil/ paclitaxel, the group of CT26 mixed with 3T3-Snail showed resistance while other groups did not.

On the other hand, our study showed that CCL1, CCL21, and CXCL1 expressed more in 3T3-Snail cell lines than 3T3 and 3T3-mock. Our previous studies have mentioned that CCL21 facilitated chemoresistance in CRC [25]. Nowadays, evidences revealed that CCL1 induced anti-apoptosis and proliferation in murine thymic lymphoma cell lines treated with dexamethasone [10]. As expected, we found that the expression of MDR1 increased in CT26 after co-cultured with 3T3-Snail or treated with CCL1. Furthermore, evidences raised that TGF- $\beta$ and NF- $\mathrm{kB}$ signaling pathways participated in drug resistance in different cancers [26,27]. Our results showed that CCL1 significantly phosphorylated ERK, Smad2, AKT, and NF-kB and the phosphorylation could be reversed by SB431542 and BAY11-7081. However, the mechanisms of those signaling pathways need more evidence to be supported. These findings may indicate CCL1 as a potential agent candidate of 5-fluorouracil/paclitaxel resistance leaded by 3T3-Snail.

Thus, our study proved that Snail-expression fibroblasts were one of the crucial factors to chemoresistance in CRC. Snail in 3T3 cells could improve the proliferation of CRC cells even explored to 5-fluorouracil/ paclitaxel. CCL1 might participate in chemoresistance of CRC through TGF- $\beta$ and NF-kB signaling pathways. Our experiments indicated that the Snail-expression fibroblasts might be a promising therapeutic option for chemoresistance in CRC.

\section{Electronic Supplementary Material}

Supplementary materials are available at Cancer Research and Treatment website (http://www.e-crt.org).

\section{Conflicts of Interest}

Conflict of interest relevant to this article was not reported.

\section{Acknowledgments}

This work was funded by the National Natural Science Foundation of China (Nos.81272311, 81472643 and 81672943). 


\section{References}

1. Jemal A, Bray F, Center MM, Ferlay J, Ward E, Forman D. Global cancer statistics. CA Cancer J Clin. 2011;61:69-90.

2. Yao J, Huang A, Zheng X, Liu T, Lin Z, Zhang S, et al. 53BP1 loss induces chemoresistance of colorectal cancer cells to 5-fluorouracil by inhibiting the ATM-CHK2-P53 pathway. J Cancer Res Clin Oncol. 2017;143:419-31.

3. De Wever O, Demetter P, Mareel M, Bracke M. Stromal myofibroblasts are drivers of invasive cancer growth. Int J Cancer. 2008;123:2229-38.

4. Kalluri R. The biology and function of fibroblasts in cancer. Nat Rev Cancer. 2016;16:582-98.

5. Piera-Velazquez S, Li Z, Jimenez SA. Role of endothelial-mesenchymal transition (EndoMT) in the pathogenesis of fibrotic disorders. Am J Pathol. 2011;179:1074-80.

6. Pan R, Zhang Y, Zheng M, Zang B, Jin M. Hydroxysafflor yellow A suppresses MRC- 5 cell activation induced by TGF-beta1 by blocking TGF-beta1 binding to TbetaRII. Front Pharmacol. 2017;8:264.

7. Marsh T, Pietras K, McAllister SS. Fibroblasts as architects of cancer pathogenesis. Biochim Biophys Acta. 2013;1832:1070-8.

8. Miller MD, Krangel MS. The human cytokine I-309 is a monocyte chemoattractant. Proc Natl Acad Sci U S A. 1992;89: 2950-4.

9. Das S, Sarrou E, Podgrabinska S, Cassella M, Mungamuri SK, Feirt N, et al. Tumor cell entry into the lymph node is controlled by CCL1 chemokine expressed by lymph node lymphatic sinuses. J Exp Med. 2013;210:1509-28.

10. Spinetti G, Bernardini G, Camarda G, Mangoni A, Santoni A, Capogrossi MC, et al. The chemokine receptor CCR8 mediates rescue from dexamethasone-induced apoptosis via an ERKdependent pathway. J Leukoc Biol. 2003;73:201-7.

11. Naber HP, Drabsch Y, Snaar-Jagalska BE, ten Dijke P, van Laar T. Snail and Slug, key regulators of TGF-beta-induced EMT, are sufficient for the induction of single-cell invasion. Biochem Biophys Res Commun. 2013;435:58-63.

12. Franci C, Gallen M, Alameda F, Baro T, Iglesias M, Virtanen I, et al. Snail1 protein in the stroma as a new putative prognosis marker for colon tumours. PLoS One. 2009;4:e5595.

13. Wu Y, Zhou BP. Snail: more than EMT. Cell Adh Migr. 2010;4:199-203.

14. Liu C, Qi Y, Qiao R, Hou Y, Chan K, Li Z, et al. Detection of early primary colorectal cancer with upconversion luminescent NP-based molecular probes. Nanoscale. 2016;8:12579-87.

15. Szymczak-Workman AL, Vignali KM, Vignali DA. Verification of 2A peptide cleavage. Cold Spring Harb Protoc. 2012;
2012:255-7.

16. Longley DB, Harkin DP, Johnston PG. 5-fluorouracil: mechanisms of action and clinical strategies. Nat Rev Cancer. 2003;3:330-8.

17. Brito DA, Yang Z, Rieder CL. Microtubules do not promote mitotic slippage when the spindle assembly checkpoint cannot be satisfied. J Cell Biol. 2008;182:623-9.

18. Porter AG, Janicke RU. Emerging roles of caspase-3 in apoptosis. Cell Death Differ. 1999;6:99-104.

19. Van Snick J, Houssiau F, Proost P, Van Damme J, Renauld JC. I-309/ T cell activation gene- 3 chemokine protects murine $\mathrm{T}$ cell lymphomas against dexamethasone-induced apoptosis. J Immunol. 1996;157:2570-6.

20. Romano M, De Francesco F, Gringeri E, Giordano A, Ferraro GA, Di Domenico M, et al. Tumor microenvironment versus cancer stem cells in cholangiocarcinoma: synergistic effects? J Cell Physiol. 2016;231:768-76.

21. Hu Y, Yan C, Mu L, Huang K, Li X, Tao D, et al. Fibroblastderived exosomes contribute to chemoresistance through priming cancer stem cells in colorectal cancer. PLoS One. 2015;10:e0125625.

22. Lotti F, Jarrar AM, Pai RK, Hitomi M, Lathia J, Mace A, et al. Chemotherapy activates cancer-associated fibroblasts to maintain colorectal cancer-initiating cells by IL-17A. J Exp Med. 2013;210:2851-72.

23. Stanisavljevic J, Loubat-Casanovas J, Herrera M, Luque T, Pena R, Lluch A, et al. Snail1-expressing fibroblasts in the tumor microenvironment display mechanical properties that support metastasis. Cancer Res. 2015;75:284-95.

24. Mukaida N, Sasaki S. Fibroblasts, an inconspicuous but essential player in colon cancer development and progression. World J Gastroenterol. 2016;22:5301-16.

25. Lu LL, Chen XH, Zhang G, Liu ZC, Wu N, Wang H, et al. CCL21 facilitates chemoresistance and cancer stem cell-Like properties of colorectal cancer cells through AKT/GSK-3beta / Snail signals. Oxid Med Cell Longev. 2016;2016:5874127.

26. Nunes JJ, Pandey SK, Yadav A, Goel S, Ateeq B. Targeting NF-kappa B signaling by artesunate restores sensitivity of castrate-resistant prostate cancer cells to antiandrogens. Neoplasia. 2017;19:333-45.

27. Song Y, Kim JS, Choi EK, Kim J, Kim KM, Seo HR. TGF- $\beta$ independent CTGF induction regulates cell adhesion mediated drug resistance by increasing collagen I in HCC. Oncotarget. 2017;8:21650-62. 